Bull. Austral. Math. Soc.

VOL. 51 (1995) [301-308]

\title{
EXPANSIVITY OF SEMI-HYPERBOLIC LIPSCHITZ MAPPINGS
}

\author{
P. Diamond, P. Kloeden, V. Kozyakin and A. Pokrovskil
}

\begin{abstract}
Semi-hyperbolic dynamical systems generated by Lipschitz mappings are shown to be exponentially expansive, locally at least, and explicit rates of expansion are determined. The result is applicable to nonsmooth noninvertible systems such as those with hysteresis effects as well as to classical systems involving hyperbolic diffeomorphisms.
\end{abstract}

\section{INTRODUCTION}

Complicated dynamical behaviour is often a consequence of the expansivity of a dynamical system, a concept that has been formulated quite simply: no two distinct trajectories can remain forever within a prescribed threshhold of each other. Expansive systems have, not surprisingly, been investigated intensively for many years (see $[2,3$, $4,5,6]$ ), particularly in regard to their entropy and structural stability.

Frequently considered examples of expansive systems involve hyperbolic diffeomorphisms, such as Smale's horseshoe mapping, in which the rate of separation of trajectories is in fact exponential, at least locally; information about this rate is very useful in both theoretical and numerical calculations of specific examples. Hyperbolicity, however, imposes far more structure on a dynamical system than is required for exponential expansivity, for example the continuity of the splitting and the existence of invariant stable and unstable manifolds. In addition, there are many important and interesting dynamical systems which lack the smoothness or invertibility of diffeomorphisms, such as those with hysteresis effects or those that arise in the finite-arithmetic computer simulation of dynamical systems. These are the subject of other investigations by the authors and will appear elsewhere.

Many useful properties of hyperbolic diffeomorphisms are retained by the semihyperbolicity mappings introduced to study local diffeomorphisms (see also Anosov [1] where a related concept was discussed). In our work appearing elsewhere, shadowinglike properties and numerical approximation are of primary interest. Here it will be

\section{Received 23rd May, 1995}

P. Diamond, P. Kloeden and A. Pokrovskii were supported by the Australian Research Council Grant A 89132609 , V.Kozyatin was partially supported by the Russian Foundation for Fundamental Research Grant 93-01-00884.

Copyright Clearance Centre, Inc. Serial-fee code: 0004-9729/95 \$A2.00+0.00. 
shown that semi-hyperbolicity on a compact set not only implies exponential expansivity, but also provides an explicit estimate of the rate of expansion. The mappings considered need only be Lipschitz and invariance of the compact set is not required.

Definitions of expansivity and exponential expansivity are given in the next section and that of semi-hyperbolicity for Lipschitz mappings in Section 3. The main result of the paper is stated in Section 4 and then proved in Section 5.

\section{Expansive Dynamical Systems}

A mapping $f$ of a metric space $(\Omega, \rho)$ into itself is considered to generate a (discrete-time) dynamical system on the state space $\Omega$ with trajectories defined recursively by

$$
x_{n}=f\left(x_{n-1}\right)=f^{n}\left(x_{0}\right)
$$

for all appropriate integers $n$. The dynamical system is said to be $\xi$-expansive [6] if $f: \Omega \mapsto \Omega$ is a homeomorphism and the inequalities $\rho\left(f^{n}\left(x_{0}\right), f^{n}\left(y_{0}\right)\right) \leqslant \xi$ for $n=$ $0, \pm 1, \pm 2, \ldots$ imply that $x_{0}=y_{0}$, that is if any trajectories remaining always within a threshhold $\xi$ of each other are identical. Usually the space $(\Omega, \rho)$ is also assumed to be compact as it is this together with expansivity that gives rise to complicated dynamical behaviour.

In expansive systems distinct trajectories often separate exponentially fast, at least locally. For example, for the shift mapping $T$ on the compact metric space $\left(\Sigma_{2}, \rho_{2}\right)$ of bi-infinite binary sequences $x=\left\{x_{i}\right\}_{i=-\infty}^{+\infty}$, that is with $x_{i} \in\{0,1\}$ and $T\left(x_{i}\right)=x_{i+1}$, it can be shown that

$$
\rho_{2}\left(T^{n}(\mathbf{x}), T^{n}(\mathbf{y})\right) \geqslant 2^{n-1} \rho_{2}(T(\mathbf{x}), T(\mathbf{y}))
$$

for $n=-n_{-}, \ldots,-1,0,1, \ldots, n_{+}$whenever

$$
\rho_{2}(x, y)=\sum_{i=-\infty}^{+\infty} 2^{-|i|}\left|x_{i}-y_{i}\right| \leqslant \xi<1
$$

where $n_{-}, n_{+}$are the largest integers such that $\rho_{2}\left(T^{n}(\mathbf{x}), T^{n}(y)\right) \leqslant \xi$ for $n=-n_{-}$, $\ldots,-1,0,1, \ldots, n_{+}$. This example is of fundamental interest because the shift mapping $T$ is conjugate to the hyperbolic diffeomorphism of the Smale horseshoe system. It motivates the following definition.

Let $|\cdot|$ denote a fixed norm on $\mathbb{R}^{d}$ and $V_{\varepsilon}(S)$ the open $\varepsilon$-neighbourhood of a nonempty subset $S \subset \mathbb{R}^{d}$. Also let $\mathcal{X}$ be an open bounded subset of $\mathbb{R}^{d}, K$ a compact subset of $\mathcal{X}$ and $r$ a positive constant. 
A continuous mapping $f: \mathcal{X} \mapsto \mathbb{R}^{d}$ is said to be exponentially expansive on $K$ with exponent $r$ if there exist $\xi, c>0$ such that $\vee_{\xi}(K) \subseteq \mathcal{X}$ and either

$$
\left|x_{n}-y_{n}\right| \geqslant c r^{n}\left|x_{0}-y_{0}\right|, \quad \text { for each } n=1,2, \ldots, n_{+}
$$

or

$$
\left|x_{n}-y_{n}\right| \geqslant c r^{-n}\left|x_{0}-y_{0}\right|, \quad \text { for each } n=-1,-2, \ldots,-n_{-}
$$

holds, or both groups of inequalities hold, for any (finite) trajectories

$$
\mathbf{x}=\left\{x_{-n_{-}}, \ldots, x_{-1}, x_{0}, x_{1}, \ldots, x_{n_{+}}\right\}, \quad \mathbf{y}=\left\{y_{-n_{-}}, \ldots, y_{-1}, y_{0}, y_{1}, \ldots, y_{n_{+}}\right\}
$$

satisfying $\mathrm{x} \subseteq K$ and $\left|y_{i}-x_{i}\right| \leqslant \xi$ for $n=-n_{-}, \ldots, 0, \ldots, n_{+}$.

\section{SEMI-HYPERBOLIC SYSTEMS IN $\mathbb{R}^{d}$}

Let $\mid \cdot$ be a fixed norm on $\mathbb{R}^{d}$ and $\mathcal{X}$ an open bounded subset of $\mathbb{R}^{d}$. Denote by $\mathcal{L}=\mathcal{L}\left(\mathcal{X}, \mathbb{R}^{d}\right)$ the set of Lipschitz mappings $f: \mathcal{X} \mapsto \mathbb{R}^{d}$.

A four-tuple $\mathbf{s}=\left(\lambda_{s}, \lambda_{u}, \mu_{s}, \mu_{u}\right)$ of nonnegative real numbers is called a split if

$$
\lambda_{s}<1<\lambda_{u}
$$

and

$$
\left(1-\lambda_{s}\right)\left(\lambda_{u}-1\right)>\mu_{s} \mu_{u} .
$$

Clearly, for any given $\lambda_{s}, \lambda_{u}$ satisfying (3) the four-tuple $s$ is a split if the product $\mu_{s} \mu_{u}$ is small enough.

Let $\mathbf{s}=\left(\lambda_{s}, \lambda_{u}, \mu_{s}, \mu_{u}\right)$ be a split and $K$ a compact subset of $\mathcal{X}$. A mapping $f \in \mathcal{L}\left(\mathcal{X}, \mathbb{R}^{d}\right)$ is said to be s-semi-hyperbolic on the set $K$ if there exist positive real numbers $k, \delta$ such that for each $x \in K$ there exists a splitting (decomposition)

$$
\mathbb{R}^{d}=E_{x}^{s} \oplus E_{x}^{u}
$$

with corresponding projectors $P_{x}^{s}$ and $P_{z}^{u}$ satisfying the following three properties:

SH0. $\operatorname{dim}\left(E_{x}^{s}\right)=\operatorname{dim}\left(E_{f(x)}^{s}\right) \quad$ if $\quad x, f(x) \in K$.

SH1. $\sup _{x \in K}\left\{\left|P_{x}^{s}\right|,\left|P_{x}^{u}\right|\right\} \leqslant k$.

SH2. The inclusion

$$
x+u+v \in \mathcal{X}
$$


and the inequalities

$$
\begin{aligned}
& \left|P_{f(x)}^{z}(f(x+u+v)-f(x+\tilde{u}+v))\right| \leqslant \lambda_{\mathrm{s}}|u-\tilde{u}|, \\
& \left|P_{f(x)}^{z}(f(x+u+v)-f(x+u+\tilde{v}))\right| \leqslant \mu_{\mathrm{a}}|v-\tilde{v}|, \\
& \left|P_{f(x)}^{u}(f(x+u+v)-f(x+\tilde{u}+v))\right| \leqslant \mu_{u}|u-\tilde{u}|, \\
& \left|P_{f(x)}^{u}(f(x+u+v)-f(x+u+\tilde{v}))\right| \geqslant \lambda_{u}|v-\tilde{v}| .
\end{aligned}
$$

hold for all $x \in K$ with $f(x) \in K$ and all $u, \widetilde{u} \in E_{x}^{s}, v, \tilde{v} \in E_{x}^{u}$ such that $|u|,|\widetilde{u}|,|v|,|\widetilde{v}| \leqslant \delta$.

The first three inequalities in SH2 are just local Lipschitz conditions on the projections of the mapping $f$ while the last one is an expansivity condition which implies a local invertibility in the unstable direction of $f$. Note that continuity in $x$ of the splitting subspaces $E_{x}^{s}, E_{x}^{u}$ or of the projectors $P_{x}^{s}, P_{x}^{u}$ is not assumed here, nor is invariance of the splitting subspaces, as is the case in the definition of hyperbolicity (of a diffeomorphism). A hyperbolic (with respect to the Euclidean metric $|\cdot|)$ diffeomorphism on $K$ is semi-hyperbolic in the above sense as a Lipschitz mapping for an appropriate triple $(\mathrm{s}, k, \delta)$.

\section{Main Result}

Some additional notation is required to formulate the main result of the paper that a Lipschitz mapping $f$ which is semi-hyperbolic on a compact subset $K$ is exponentially expansive on $K$ and, moreover, that the coefficients of exponential expansivity can be expressed in terms of the constants of the split $\mathbf{s}$ and the semi-hyperbolicity constants $k, \delta$.

For a split $\mathbf{s}=\left(\lambda_{s}, \lambda_{u}, \mu_{s}, \mu_{u}\right)$ define the matrix

$$
M(s)=\left(\begin{array}{cc}
\lambda_{s} & \mu_{s} \\
\frac{\mu_{u}}{\lambda_{u}} & \frac{1}{\lambda_{u}}
\end{array}\right)
$$

From inequalities (3), (4) its spectral radius

$$
\sigma(s)=\frac{1}{2}\left(\left(\frac{1}{\lambda_{u}}+\lambda_{s}\right)+\sqrt{\left(\frac{1}{\lambda_{u}}-\lambda_{s}\right)^{2}+\frac{4 \mu_{s} \mu_{u}}{\lambda_{u}}:}\right)
$$

satisfies

$$
\sigma(\mathbf{s})<1
$$


and, since the entries of the matrix $M(\mathrm{~s})$ are positive, it follows by the Perron-Frobenius theorem that $\sigma(s)$ is the maximal eigenvalue of $M(s)$ and that the corresponding eigenvector has positive coordinates. This eigenvector will be written here as $(1, \gamma(\mathbf{s}))^{T}$, where

$$
\gamma(s)=\frac{1}{2 \mu_{s}}\left(\left(\frac{1}{\lambda_{u}}-\lambda_{s}\right)+\sqrt{\left(\frac{1}{\lambda_{u}}-\lambda_{s}\right)^{2}+\frac{4 \mu_{s} \mu_{u}}{\lambda_{u}}:}\right) .
$$

The main result of the paper is then

ThEorem 1. Let $f \in \mathcal{L}\left(\mathcal{X}, \mathbb{R}^{d}\right)$ be s-semi-hyperbolic in $K \subseteq \mathcal{X}$. Then it is exponentially expansive in $K$ with exponent $r=\sigma(s)^{-1}$.

\section{Proof of Theorem 1}

In fact, we shall show expansivity with the explicit form for the constants:

$$
c=\frac{1}{2} k^{-1} \min \left\{\gamma(\mathrm{s}), \gamma(\mathrm{s})^{-1}\right\} \quad \text { and } \quad \xi=k^{-1} \delta
$$

Three lemmata will form the basis of the proof. Since $\mathbf{s}$ is fixed write $M=M(\mathbf{s}), \gamma=$ $\gamma(\mathbf{s})$ and $\sigma=\sigma(\mathbf{s})$, so

$$
M\left(\begin{array}{l}
1 \\
\gamma
\end{array}\right)=\left(\begin{array}{cc}
\lambda_{s} & \mu_{s} \\
\frac{\mu_{u}}{\lambda_{u}} & \frac{1}{\lambda_{u}}
\end{array}\right)\left(\begin{array}{l}
1 \\
\gamma
\end{array}\right)=\sigma\left(\begin{array}{l}
1 \\
\gamma
\end{array}\right)
$$

and introduce a new norm $\|\cdot\|$ on $\mathbb{R}^{2}$ defined by

$$
\left\|\left(y_{1}, y_{2}\right)\right\|=\max \left\{\left|y_{1}\right|, \gamma\left|y_{2}\right|\right\}
$$

Clearly, the corresponding norm $\|M\|$ of the linear operator with the matrix (6) coincides with the spectral radius $\sigma$ of $M$, so $\|M\|=\sigma<1$ by (7), with $\|M \mathbf{u}\| \leqslant \sigma\|\mathbf{u}\|$ for all $u \in \mathbb{R}^{2}$.

LEMMA 1. There exists a constant $\zeta>0$ such that

$$
\zeta \max \left\{\left|y_{1}\right|,\left|y_{2}\right|\right\} \leqslant\left\|\left(y_{1}, y_{2}\right)\right\| \leqslant \zeta \max \left\{\gamma, \gamma^{-1}\right\} \max \left\{\left|y_{1}\right|,\left|y_{2}\right|\right\}
$$

Proof: It suffices to define $\zeta=1$ if $\gamma \geqslant 1$ and $\zeta=\gamma$ if $\gamma<1$.

LEMMA 2. There exists a constant $\eta>0$ such that

$$
\eta|z| \leqslant\left\|\left(\left|P_{x}^{\lrcorner} z\right|,\left|P_{x}^{u} z\right|\right)\right\| \leqslant 2 \eta k \max \left\{\gamma, \gamma^{-1}\right\}|z|
$$


for all $x \in K$ and $z \in \mathbb{R}^{d}$.

Proof: By Lemma 1 it suffices to check that the inequalities

$$
\max \left\{\left|P_{x}^{s} z\right|,\left|P_{x}^{u} z\right|\right\} \leqslant k|z| \leqslant 2 k \max \left\{\left|P_{z}^{s} z\right|,\left|P_{x}^{u} z\right|\right\}
$$

hold for all $x \in K$ and $z \in \mathbb{R}^{d}$. The inequality on the left with $\eta=\zeta / 2$ follows from the semi-hyperbolicity property $\mathrm{SH} 1$, while that on the right is just

$$
|z|=\left|P_{x}^{s} z+P_{x}^{u} z\right| \leqslant\left|P_{x}^{s} z\right|+\left|P_{x}^{u} z\right| \leqslant 2 \max \left\{\left|P_{x}^{s} z\right|,\left|P_{x}^{u} z\right|\right\}
$$

multipled through by $k$.

For the remaining lemma note that semi-hyperbolicity does not require the sets $K$ and $\mathcal{X}$ to be invariant under the mapping $f$.

LEMma 3. Suppose that $x, f(x), f^{2}(x) \in K$ and $y, f(y), f^{2}(y) \in \mathcal{X}$ with

$$
|x-y|,|f(x)-f(y)|,\left|f^{2}(x)-f^{2}(y)\right|<\delta
$$

Then at least one of the following pair of inequalities

$$
\begin{array}{r}
\left\|\left(\left|P_{x}^{s} r_{0}\right|,\left|P_{x}^{u} r_{0}\right|\right)\right\| \geqslant \sigma^{-1}\left\|\left(\left|P_{f(x)}^{s} r_{1}\right|,\left|P_{f(x)}^{u} r_{1}\right|\right)\right\|, \\
\left\|\left(\left|P_{f^{2}(x)}^{s} r_{2}\right|,\left|P_{f^{2}(x)}^{u} r_{2}\right|\right)\right\|
\end{array}
$$

holds, where

$$
r_{0}=x-y, \quad r_{1}=f(x)-f(y), \quad r_{2}=f^{2}(x)-f^{2}(y) .
$$

ProOF: By Property SH2 of semi-hyperbolicity and the above assumptions

$$
\begin{aligned}
& \left|P_{f(x)}^{s} r_{1}\right| \leqslant \lambda_{0}\left|P_{x}^{s} r_{0}\right|+\mu_{0}\left|P_{x}^{u} r_{0}\right| \\
& \left|P_{f^{2}(x)}^{u} r_{2}\right| \geqslant \lambda_{u}\left|P_{f(x)}^{u} r_{1}\right|-\mu_{u}\left|P_{f(x)}^{\prime} r_{1}\right|
\end{aligned}
$$

or, what is the same,

$$
\begin{aligned}
& \left|P_{f(x)}^{u} r_{1}\right| \leqslant \lambda_{0}\left|P_{x}^{*} r_{0}\right|+\mu_{0}\left|P_{x}^{u} r_{0}\right| \\
& \left|P_{f(x)}^{u} r_{1}\right| \leqslant \frac{\mu_{u}}{\lambda_{u}}\left|P_{f(x)}^{z} r_{1}\right|+\frac{1}{\lambda_{u}}\left|P_{f^{2}(x)}^{u} r_{2}\right| .
\end{aligned}
$$

Suppose that the lemma is not true, that is, both

$$
\begin{array}{r}
\left\|\left(\left|P_{x}^{u} r_{0}\right|,\left|P_{x}^{u} r_{0}\right|\right)\right\|<\sigma^{-1}\left\|\left(\left|P_{f(x)}^{e} r_{1}\right|,\left|P_{f(x)}^{u} r_{1}\right|\right)\right\|, \\
\left\|\left(\left|P_{f^{2}(x)}^{u} r_{2}\right|,\left|P_{f^{2}(x)}^{u} r_{2}\right|\right)\right\|<\sigma^{-1}\left\|\left(\left|P_{f(x)}^{e} r_{1}\right|,\left|P_{f(x)}^{u} r_{1}\right|\right)\right\|
\end{array}
$$


are true. Then inequality (13) implies that

$$
\left\|\left(\left|P_{f(x)^{r_{1}}}^{r_{1}}\right|,\left|P_{f^{2}(x)}^{u} r_{2}\right|\right)\right\|<\sigma^{-1}\left\|\left(\left|P_{f(x)}^{s} r_{1}\right|,\left|P_{f(x)}^{u} r_{1}\right|\right)\right\|,
$$

while from (10), (12) and (9) it follows that

$$
\left|P_{f(x)}^{s} r_{1}\right|<\left\|\left(\left|P_{f(x)}^{s} r_{1}\right|,\left|P_{f(x)}^{u} r_{1}\right|\right)\right\|
$$

and, analogously, from (11), (14) and $\sigma=\|M\|$, that

$$
\gamma\left|P_{f(x)}^{u} r_{1}\right|<\left\|\left(\left|P_{f(x)}^{s} r_{1}\right|,\left|P_{f(x)}^{u} r_{1}\right|\right)\right\| .
$$

Now inequalities (15) and (16) imply that

$$
\left\|\left(\left|P_{f(x)}^{s} r_{1}\right|,\left|P_{f(x)}^{u} r_{1}\right|\right)\right\|<\left\|\left(\left|P_{f(x)}^{s} r_{1}\right|,\left|P_{f(x)}^{u} r_{1}\right|\right)\right\|,
$$

which is a contradiction and the lemma is proved.

To complete the proof of the theorem consider two trajectories

$$
\mathbf{x}=\left\{x_{-n_{-}}, \ldots, x_{-1}, x_{0}, x_{1}, \ldots, x_{n_{+}}\right\}, \quad \mathbf{y}=\left\{y_{-n_{-}}, \ldots, y_{-1}, y_{0}, y_{1}, \ldots, \dot{y}_{n_{+}}\right\}
$$

satisfying $\mathrm{x} \subseteq K, \mathrm{y} \subseteq \mathcal{X}$ and $\left|y_{n}-x_{n}\right| \leqslant \xi$ for $n=-n_{-}, \ldots, n_{+}$and write

$$
r_{n}=x_{n}-y_{n}, \quad \nu_{n}=\left\|\left(\left|P_{f\left(x_{n}\right)}^{s} r_{n}\right|,\left|P_{f\left(x_{n}\right)}^{u} r_{n}\right|\right)\right\|
$$

for $n=-n_{-}, \ldots, n_{+}$. From Lemma 3 it then follows for $n=-n_{-}+1, \ldots, n_{+}-1$ that at least one of the inequalities

$$
\nu_{n-1}>\sigma^{-1} \nu_{n}, \quad \nu_{n+1}>\sigma^{-1} \nu_{n}
$$

holds, from which in turn the inequalities (1) or (2) are obtained by Lemma 3 again. Indeed, if

$$
\nu_{-1}>\sigma^{-1} \nu_{0}
$$

then it follows from (17) that

$$
\nu_{n-1}>\sigma^{-1} \nu_{n}, \quad \text { for } n=-n_{-}+1, \ldots,-1,0,
$$

while if $\nu_{1}>\sigma^{-1} \nu_{0}$ then the inequalities (1) also follow by Lemma 3 .

This completes the proof of Theorem 1. 
REMARK. As is seen from the proof of Theorem 1, in questions about expansivity, the conditions SHO - SH2 of the definition of semi-hyperbolicity are superfluous. By the same way can be proved the following stronger modification of Theorem 1 . Let $\mathbf{s}=\left(\lambda_{a}, \lambda_{u}, \mu_{s}, \mu_{u}\right)$ be a split, $K$ be a compact subset of $\mathcal{X}$ and $k, \delta$ be some positive real numbers.

Theorem 2. Let $f \in \mathcal{L}\left(\mathcal{X}, \mathbb{R}^{d}\right)$ and suppose for each $x \in K$ there exists a splitting (5) satisfying the property $\mathrm{SH} 1$ and the following modification of the property SH2: for all $x$ satisfying $x, f(x) \in K$ and for all $z \in \mathbb{R}^{d}$ satisfying $\left|P_{x}^{s} z\right|,\left|P_{x}^{u} z\right| \leqslant \delta$, there hold the inclusion $x+z \in \mathcal{X}$ and the inequalities

$$
\begin{aligned}
& \left|P_{f(x)}^{s}(f(x+z)-f(x))\right| \leqslant \lambda_{s}\left|P_{x}^{s} z\right|+\mu_{s}\left|P_{x}^{u} z\right| \\
& \left|P_{f(x)}^{u}(f(x+z)-f(x))\right| \geqslant-\mu_{u}\left|P_{x}^{s} z\right|+\lambda_{u}\left|P_{x}^{u} z\right|
\end{aligned}
$$

Then the mapping $f$ is exponentially expansive in $K$ with exponent $r=\sigma(s)^{-1}$ and constants (8).

\section{References}

[1] D. Anosov, 'Geodesic flows on closed Riemannian manifolds with negative curvature', Proc. Steklov Inst. Math. 90 (1967), 1-235.

[2] J. Appel, V. Lakshmikantham, N. van Minh and P.P. Zabreiko, 'A general model of evolutionary processes. Exponential dichtomy - I', Nonlinear Anal. 21 (1993), 207-218.

[3] J. Appel, V. Lakshmikantham, N. van Minh and P.P. Zabreiko, 'A general model of evolutionary processes. Exponential dichtomy - II', Nonlinear Anal. 21 (1993), 219-225.

[4] J. Palis and W. de Melo, Geometric theory of dynamical systems. An introduction (Springer-Verlag, Berlin, Heidelberg, New York, 1982).

[5] D. Reuelle, Elements of differentiable dynamics and bifurcation theory (Academic Press, Inc., Boston, 1989).

[6] W.R. Utz, 'Unstable homeomorphisms', Proc. Amer. Math. Soc. 1 (1950), 357-368.

Department of Mathematics

University ofQueensland

Queensland 4072 Australia

Institute of Information Transmission Problems Russian Academy of Sciences

19 Ermolovoy str.

Moscow 101477

Russia
Department of Computing and Mathematics

Deakin University

Geelong Vic 3217 Australia

Institute of Information Transmission Problems Russian Academy of Sciences

19 Ermolovoy str.

Moscow 101477

Russia 